\title{
Physical, Functional Properties and Cooking Quality of Mothbean [Vigna aconitifolia (Jacq.) Marechal] Varieties
}

\author{
Pallavi Badami* and B. Kasturiba \\ Department of Food Science and Nutrition, College of Community Science, \\ University of Agricultural Sciences, Dharwad, Karnataka, India \\ Corresponding author
}

\section{A B S T R A C T}

K e y w or d s
Mothbean, Physical
properties,
Functional
properties, Cooking
quality

Pulses are widely grown and consumed throughout the world and also form the important source of protein and thus continue to occupy an important place in human nutrition particularly in developing countries like India. A study was conducted to analyze the physical, functional properties and cooking quality of mothbean varieties. Significant differences were found among the varieties for all physical and functional properties. The length, breadth, hundred kernel weight, hundred kernel volume and grain density ranged from 3.67-3.99 mm, 2.20-2.40 mm, 1.61-1.96 g, 1.26-1.53 ml and 1.16-1.44 g/ml respectively. Hydration capacity, hydration index, swelling capacity, swelling index ranged from 2.31-2.75 g/100 grains, 1.25-1.52, 3.7-4.86 ml/100 grains, 2.25-3.88 on dry weight basis. Significant difference was found in germination percentage among the varieties and it ranged from 89.33-96.33 per cent, being highest in RMB-108 and lowest in BGMB-14. Cooked weight and volume of grains without soaking and with soaking ranged from 19.52-20.30 g, 19.28-20.05g, 16.33-19.66 $\mathrm{ml}$ and 14.33-17.66 ml respectively. Cooking time and solid loss of grains without soaking and with soaking ranged from 30.33-33.66 min, 19-24 min, 5.63-6.72 per cent and 5.73-6.62 per cent respectively.

\section{Introduction}

Food legumes are crops of the family Leguminasae also called Fabacae. Grain legumes used in combination with cereals are grown in both tropical and temperate regions of the world viz., Africa, Asia, West Indies and Latin America. India is the largest consumer and importer of pulses in the world and they are consumed regularly in every household at least with one meal. They enhance the protein content of cereal-based diets and improve the nutritional status of the cereal-based diets. Legumes not only add variety to human diet but also serve as an economical source of supplementary proteins for a large population in developing countries like India. Pulses play a significant role in mitigating protein malnutrition of millions of poor vegetarians in tropical and sub-tropical regions of world. Hence, legumes are considered as "poor man's meat". Legumes apart from being an important source of macro nutrients (carbohydrates, protein, fiber) and 
micronutrients (calcium, magnesium, zinc, iron, potassium, phosphorous), the health benefits of consuming legumes are also related to the amount of dietary fiber and polyphenols present in legumes. Mothbean (Vigna aconitifolia (Jacq.) Marechal) is one of the primitive pulses which is consumed throughout India. Mothbean is recognized for its twin tolerance to drought and heat. It is, therefore, the ultimate choice of the marginal and sub-marginal farmers for realization of sustained production under the harsh agroclimatic situations. Mothbean is a source of food, fodder, feed and green manuring hence, serves as a multi-purpose crop. Green pods are delicious source of vegetables. Being pulse, it is a cheap source of vegetable protein for balancing the nutritional deficiency. It is regarded as a quality pulse crop in India for its excellent protein quality, high digestibility and freedom from flatulence value compared to other pulses.

Agricultural scientists and breeders are involved in development and release of new varieties having better physical properties and functional properties which inturn influences the cooking quality. Hence the present study was undertaken with the objective to analyze the physical, functional properties and cooking quality of mothbean varieties.

\section{Materials and Methods}

Eleven mothbean varieties were procured from AICRP for dry land agriculture, Regional Agricultural Research Station, Vijayapur, University of Agricultural Sciences, Dharwad, Karnataka state in India. Among the eleven varieties, three were prereleased varieties (BGMBG-10, BGMB-11 and BGMB-14) and eight were released varieties (CZM-3, CGM-18, RMB-101, RMB108, RMB-141, RMB-408, MBS-27, KBMB1 ). The average length and breadth of the randomly picked ten grains were measured with a help of digital vernier calipers having least count $0.01 \mathrm{~mm}$ (Yadav et al., 2007). Hundred kernel weight, hundred kernel volume and grain density was assessed using the method given by Khattak et al., 2007. Hydration capacity and swelling capacity was carried out using the method given by Bishnoi and Khetarpaul (1993). Hydration index and swelling index was carried out using the method given by Khattak et al., (2007). For germination, hundred grains were placed in a petriplate on a filter paper dampened with water and the lid was closed, kept in incubator and allowed to germinate for $24 \mathrm{hrs}$. Further germinated grains were counted and expressed in percentage (Barakoti and Bains., 2007). Cooked weight, cooked volume, cooking time and solid loss of grains without and with soaking was assessed by following the procedure given by Sareepuang et al., 2008.

\section{Results and Discussion}

Physical properties i.e length, breadth, hundred kernel weight, hundred kernel volume and grain density are presented in Table 1. Length, breadth, hundred kernel weight, hundred kernel volume and grain density differed significantly $(\mathrm{p} \leq 0.01)$ among the varieties. Length ranged from 3.67-3.99 mm. Highest length was observed in RMB-408 (3.99 $\mathrm{mm})$ and least length was observed in RMB-101 (3.67 mm). Breadth varied from 2.20-2.40 mm. RMB-408 recorded highest breadth $(2.40 \mathrm{~mm})$, whereas BGMB-14 recorded least breadth $(2.20 \mathrm{~mm}$. The study conducted by Mankotia and Modgil (2003) showed slightly higher values for both length $(5.13 \mathrm{~mm})$ and breadth $(5.15 \mathrm{~mm})$ of the mothbean variety.

Hundred kernel weight ranged from 1.61-1.96 g. Highest was recorded in MBS-27 (1.96 g) and least was recorded in CGM-18 (1.61 g). Hundred kernel volume ranged from 1.26-1.53 $\mathrm{ml}$. Highest was recorded in RMB-141, MBS- 
27 and BGMBG-10 (1.53 ml) and least was recorded in RMB-408 (1.26 ml). Grain density ranged from 1.16-1.44 g/ml. Highest was recorded in RMB-408 $(1.44 \mathrm{~g} / \mathrm{ml})$ and least was recorded in KBMB-1 $(1.16 \mathrm{~g} / \mathrm{ml})$. Table 2 compares the physical properties of prereleased and released varieties. Results showed that there was no significant difference in all the parameters between prereleased and released varieties of mothbean. Released varieties registered higher values for length and breadth whereas pre-released varieties had higher values for hundred kernel weight, hundred kernel volume and grain density compared to released varieties. The study conducted by Bhokre and Joshi (2015) reported that hundred kernel weight and grain density of raw mothbean was $6.82 \mathrm{~g}$ and 0.786 $\mathrm{g} / \mathrm{ml}$. Mankotia and Modgil (2003) reported the similar results for hundred kernel weight and grain density. The reason for variation in the present study may be attributed to the varietal differences.

The functional properties of mothbean varieties are presented in Table 3. Significant differences were observed in all the functional properties $(p \leq 0.01)$. Hydration capacity was highest in CZM-3 (2.75 g/100 grains) and lowest in MBS-27 (2.31 g/100 grains). Highest hydration index was found in KBMB1 (1.52) and lowest was found in MBS-27 (1.25). Highest swelling capacity was recorded in CGM-18 (4.86 ml/100 grains) and lowest was found in BGMB-14 $(3.70 \mathrm{ml} / 100$ grains). Highest swelling index was recorded in CZM-3 (3.88) and lowest was recorded in BGMB-14 (2.25). Table 4 depicts the comparison of functional properties of prereleased and released varieties of mothbean. There was no significant difference in the functional properties between two groups. Released varieties registered higher values for hydration capacity, hydration index, swelling capacity and swelling index. The reason for this might be due to differences in the physical parameters among the varieties and agronomical conditions in which they are grown.

Figure 1 represents the germination of mothbean varieties. Germination ranged from 89.33-96.33 per cent. Highest was recorded in RMB-108 (96.33\%) and least was found in BGMB-14 (89.33\%). This is may be due to differences in the physical and functional properties of the mothbean.

Table 5 depicts the results of cooking quality of mothbean varieties. Cooking quality varied significantly among the varieties $(p \leq 0.01)$. The cooked weight of grains without soaking and with soaking ranged from 19.52-20.30 g and 19.28-20.05 g respectively. The cooked volume of grains without soaking and with soaking ranged from 16.33-19.66 $\mathrm{ml}$ and 14.33-17.66 $\mathrm{ml}$ respectively.

Cooking time of grains without soaking and with soaking ranged from 30.33-33.66 min and 19-24 min respectively. Grains without soaking took longer time to cook compared to grains with soaking.

Solid loss without soaking and with soaking ranged from 5.63-6.72 per cent and 5.73-6.62 per cent respectively. There was reduction in all the parameters after cooking. The study conducted by Bhokre and Joshi (2015) supports the results of preset study where cooking time was reduced after soaking process. The reasons attributed to this might be due to softening of starch and other solid matter while soaking and thus further decreasing the cooking time and solid loss.

Table 6 depicts the comparison of cooking quality of pre-released and released mothbean varieties. Significant difference $(p \leq 0.05)$ in solid loss was observed between grains without soaking and with soaking. 
Table.1 Physical properties of mothbean varieties

\begin{tabular}{|l|c|c|c|c|c|}
\hline Varieties & Length $(\mathrm{mm})$ & Breadth $(\mathrm{mm})$ & $\begin{array}{c}100 \text { kernel } \\
\text { weight }(\mathrm{g})\end{array}$ & $\begin{array}{c}100 \text { kernel } \\
\text { volume }(\mathrm{ml})\end{array}$ & $\begin{array}{c}\text { Bulk density } \\
(\mathrm{g} / \mathrm{ml})\end{array}$ \\
\hline & \multicolumn{5}{|c|}{ Pre-released varieties } \\
\hline BGMBG-10 & $3.91 \pm 0.15$ & $2.31 \pm 0.09$ & $1.82 \pm 0.007$ & $1.53 \pm 0.11$ & $1.19 \pm 0.09$ \\
\hline BGMB-11 & $3.85 \pm 0.18$ & $2.38 \pm 0.09$ & $1.84 \pm 0.004$ & $1.43 \pm 0.05$ & $1.28 \pm 0.05$ \\
\hline BGMB-14 & $3.77 \pm 0.17$ & $2.20 \pm 0.12$ & $1.91 \pm 0.02$ & $1.50 \pm 0.10$ & $1.28 \pm 0.07$ \\
\hline CZM-3 & $3.74 \pm 0.16$ & $2.29 \pm 0.11$ & $1.90 \pm 0.01$ & $1.46 \pm 0.11$ & $1.30 \pm 0.08$ \\
\hline CGM-18 & $3.88 \pm 0.09$ & $2.25 \pm 0.07$ & $1.61 \pm 0.002$ & $1.36 \pm 0.05$ & $1.17 \pm 0.04$ \\
\hline RMB-108 & $3.89 \pm 0.13$ & $2.36 \pm 0.16$ & $1.76 \pm 0.001$ & $1.36 \pm 0.05$ & $1.29 \pm 0.05$ \\
\hline RMB-408 & $3.99 \pm 0.10$ & $2.40 \pm 0.10$ & $1.81 \pm 0.006$ & $1.26 \pm 0.11$ & $1.44 \pm 0.12$ \\
\hline RMB-141 & $3.74 \pm 0.13$ & $2.36 \pm 0.06$ & $1.79 \pm 0.03$ & $1.53 \pm 0.11$ & $1.17 \pm 0.10$ \\
\hline RMB-101 & $3.67 \pm 0.08$ & $2.21 \pm 0.07$ & $1.79 \pm 0.01$ & $1.50 \pm 0.10$ & $1.20 \pm 0.07$ \\
\hline MBS-27 & $3.96 \pm 0.09$ & $2.37 \pm 0.08$ & $1.96 \pm 0.06$ & $1.53 \pm 0.11$ & $1.28 \pm 0.12$ \\
\hline KBMB-1 & $3.76 \pm 0.24$ & $2.29 \pm 0.12$ & $1.67 \pm 0.02$ & $1.43 \pm 0.05$ & $1.16 \pm 0 . .04$ \\
\hline Mean \pm SD & $3.83 \pm 0.17$ & $2.31 \pm 0.11$ & $1.81 \pm 0.10$ & $1.44 \pm 0.11$ & $1.25 \pm 0.10$ \\
\hline S. Em. \pm & 0.04 & 0.03 & 0.01 & 0.05 & 0.04 \\
\hline C.D & $0.12 * *$ & $0.08 * *$ & $0.04 * *$ & $0.16 *$ & $0.14 *$ \\
\hline F value & 4.51 & 4.55 & 52.90 & 2.44 & 2.87 \\
\hline
\end{tabular}

Note: Values are mean of three replications, S. Em.: Standard Error of Mean, C.D @ 1\%: Critical Difference, *Significant @ 5\%,**Significant @ 1\%

Table.2 Mean physical properties of pre-released and released mothbean varieties

\begin{tabular}{|l|c|c|c|c|c|}
\hline \multicolumn{1}{|c|}{ Varieties } & Length $(\mathrm{mm})$ & Breadth $(\mathrm{mm})$ & $\begin{array}{c}100 \text { kernel } \\
\text { weight }(\mathrm{g})\end{array}$ & $\begin{array}{c}100 \text { kernel } \\
\text { volume }(\mathrm{ml})\end{array}$ & $\begin{array}{c}\text { Bulk density } \\
(\mathrm{g} / \mathrm{ml})\end{array}$ \\
\hline $\begin{array}{l}\text { Pre-released } \\
\text { varieties }\end{array}$ & $3.83 \pm 0.17$ & $2.30 \pm 0.12$ & $1.84 \pm 0.04$ & $1.48 \pm 0.09$ & $1.25 \pm 0.07$ \\
\hline $\begin{array}{l}\text { Released } \\
\text { varieties }\end{array}$ & $3.84 \pm 0.17$ & $2.31 \pm 0.11$ & $1.79 \pm 0.11$ & $1.43 \pm 0.12$ & $1.26 \pm 0.11$ \\
\hline 't' value & $\mathrm{NS}$ & $\mathrm{NS}$ & $\mathrm{NS}$ & $\mathrm{NS}$ & $\mathrm{NS}$ \\
\hline
\end{tabular}

Note: Values are mean of three replications, NS-Non significant 
Table.3 Functional properties of mothbean varieties

\begin{tabular}{|l|c|c|c|c|}
\hline \multicolumn{1}{|c|}{ Varieties } & $\begin{array}{c}\text { Hydration capacity } \\
\text { (g/100 grains) }\end{array}$ & Hydration index & $\begin{array}{c}\text { Swelling capacity (ml/ } \\
100 \text { grains })\end{array}$ & Swelling index \\
\hline BGMBG-10 & $2.44 \pm 0.007$ & $1.36 \pm 0.03$ & $3.80 \pm 0.51$ & $2.47 \pm 0.24$ \\
\hline BGMB-11 & $2.64 \pm 0.05$ & $1.41 \pm 0.03$ & $4.76 \pm 0.55$ & $3.04 \pm 0.31$ \\
\hline BGMB-14 & $2.53 \pm 0.03$ & $1.30 \pm 0.01$ & $3.70 \pm 0.51$ & $2.25 \pm 0.23$ \\
\hline & & Released varieties & & \\
\hline CZM-3 & $2.75 \pm 0.06$ & $1.37 \pm 0.03$ & $4.10 \pm 0.51$ & $3.88 \pm 0.19$ \\
\hline CGM-18 & $2.34 \pm 0.03$ & $1.39 \pm 0.02$ & $4.86 \pm 0.46$ & $3.31 \pm 0.05$ \\
\hline RMB-108 & $2.52 \pm 0.06$ & $1.33 \pm 0.04$ & $4.23 \pm 0.55$ & $2.95 \pm 0.35$ \\
\hline RMB-408 & $2.55 \pm 0.10$ & $1.40 \pm 0.09$ & $4.23 \pm 0.63$ & $2.96 \pm 0.55$ \\
\hline RMB-141 & $2.42 \pm 0.01$ & $1.36 \pm 0.03$ & $4.13 \pm 0.63$ & $2.70 \pm 0.50$ \\
\hline RMB-101 & $2.50 \pm 0.09$ & $1.39 \pm 0.04$ & $3.90 \pm 0.51$ & $2.71 \pm 0.24$ \\
\hline MBS-27 & $2.31 \pm 0.05$ & $1.25 \pm 0.009$ & $4.43 \pm 0.05$ & $2.83 \pm 0.14$ \\
\hline KBMB-1 & $2.63 \pm 0.10$ & $1.52 \pm 0.06$ & $4.20 \pm 0.60$ & $2.87 \pm 0.48$ \\
\hline Mean \pm SD & $2.51 \pm 0.13$ & $1.37 \pm 0.07$ & $4.39 \pm 0.78$ & $2.91 \pm 0.50$ \\
\hline S. Em. \pm & 0.03 & 0.02 & 0.30 & 0.19 \\
\hline C.D @ 1\% & $0.10 * *$ & $0.07 * *$ & $0.89 * *$ & $0.57 * *$ \\
\hline F value & 11.54 & 7.17 & 4.83 & 4.83 \\
\hline
\end{tabular}

Note: Values are mean of three replications, S. Em.: Standard Error of Mean, C. D: Critical Difference, **Significant @ 1\%

Table.4 Mean functional properties of pre-released and released mothbean varieties

\begin{tabular}{|l|c|c|c|c|}
\hline \multicolumn{1}{|c|}{ Varieties } & $\begin{array}{c}\text { Hydration capacity } \\
\text { (g/100 grains) }\end{array}$ & $\begin{array}{c}\text { Hydration } \\
\text { index }\end{array}$ & $\begin{array}{c}\text { Swelling capacity } \\
\text { (ml/100 grains) }\end{array}$ & $\begin{array}{c}\text { Swelling } \\
\text { index }\end{array}$ \\
\hline $\begin{array}{l}\text { Pre-released } \\
\text { varieties }\end{array}$ & $2.50 \pm 0.09$ & $1.35 \pm 0.05$ & $4.08 \pm 0.68$ & $2.59 \pm 0.41$ \\
\hline $\begin{array}{l}\text { Released } \\
\text { varieties }\end{array}$ & $2.54 \pm 0.15$ & $1.38 \pm 0.08$ & $4.51 \pm 0.80$ & $3.03 \pm 0.48$ \\
\hline 't' value & $\mathrm{NS}$ & $\mathrm{NS}$ & $\mathrm{NS}$ & $\mathrm{NS}$ \\
\hline
\end{tabular}

Note: Values are mean of three replications, NS-Non Significant 
Table.5 Cooking quality of mothbean varieties

\begin{tabular}{|c|c|c|c|c|c|c|c|c|}
\hline \multirow[t]{2}{*}{ Varieties } & \multicolumn{4}{|c|}{ Cooked grains without soaking } & \multicolumn{4}{|c|}{ Cooked grains with soaking } \\
\hline & $\begin{array}{c}\text { Cooked } \\
\text { weight } \\
\text { (g) }\end{array}$ & $\begin{array}{c}\text { Cooked } \\
\text { volume } \\
(\mathbf{m l})\end{array}$ & $\begin{array}{c}\text { Cooking } \\
\text { time } \\
(\mathrm{min})\end{array}$ & $\begin{array}{l}\text { Solid } \\
\text { loss } \\
(\%)\end{array}$ & $\begin{array}{c}\text { Cooked } \\
\text { weight } \\
\text { (g) }\end{array}$ & $\begin{array}{l}\text { Cooked } \\
\text { volume } \\
(\mathrm{ml})\end{array}$ & $\begin{array}{l}\text { Cooking } \\
\text { time } \\
\text { (min) }\end{array}$ & $\begin{array}{l}\text { Solid } \\
\text { loss } \\
(\%)\end{array}$ \\
\hline \multicolumn{9}{|c|}{ Pre-released varieties } \\
\hline $\begin{array}{l}\text { BGMBG- } \\
10\end{array}$ & $\begin{array}{c}20.30 \pm \\
0.05\end{array}$ & $\begin{array}{c}18.33 \pm \\
0.57\end{array}$ & $\begin{array}{c}31.66 \pm \\
0.56\end{array}$ & $\begin{array}{c}6.65 \pm \\
0.07\end{array}$ & $\begin{array}{c}20.05 \pm \\
0.09\end{array}$ & $\begin{array}{c}16.33 \pm \\
0.57\end{array}$ & $\begin{array}{c}21.33 \pm \\
1.52\end{array}$ & $\begin{array}{c}6.57 \pm \\
0.07\end{array}$ \\
\hline $\begin{array}{l}\text { BGMB- } \\
11\end{array}$ & $\begin{array}{c}19.94 \pm \\
0.16\end{array}$ & $\begin{array}{c}16.33 \pm \\
0.57\end{array}$ & $\begin{array}{c}33.33 \pm \\
0.58\end{array}$ & $\begin{array}{c}5.91 \pm \\
0.03\end{array}$ & $\begin{array}{c}19.69 \pm \\
0.14\end{array}$ & $\begin{array}{c}14.33 \pm \\
0.57\end{array}$ & $\begin{array}{c}24.00 \pm \\
1.00\end{array}$ & $\begin{array}{c}5.94 \pm \\
0.10\end{array}$ \\
\hline $\begin{array}{l}\text { BGMB- } \\
14\end{array}$ & $\begin{array}{c}19.52 \pm \\
0.19\end{array}$ & $\begin{array}{c}18.33 \pm \\
0.56\end{array}$ & $\begin{array}{c}31.66 \pm \\
0.57\end{array}$ & $\begin{array}{c}6.72 \pm \\
0.07\end{array}$ & $\begin{array}{c}19.28 \pm \\
0.22\end{array}$ & $\begin{array}{c}16.33 \pm \\
0.57\end{array}$ & $\begin{array}{c}20.66 \pm \\
0.57\end{array}$ & $\begin{array}{c}6.62 \pm \\
0.07\end{array}$ \\
\hline \multicolumn{9}{|c|}{ Released varieties } \\
\hline CZM-3 & $\begin{array}{l}20.1 \pm \\
0.08\end{array}$ & $\begin{array}{c}18.66 \pm \\
0.56\end{array}$ & $\begin{array}{c}30.33 \pm \\
0.57\end{array}$ & $\begin{array}{c}5.89 \pm \\
0.09\end{array}$ & $\begin{array}{c}19.67 \pm \\
0.10\end{array}$ & $\begin{array}{c}16.66 \pm \\
0.57\end{array}$ & $\begin{array}{c}19.33 \pm \\
0.57\end{array}$ & $\begin{array}{c}5.92 \pm \\
0.10\end{array}$ \\
\hline CGM-18 & $\begin{array}{c}19.84 \pm \\
0.04\end{array}$ & $\begin{array}{c}17.33 \pm \\
0.58\end{array}$ & $\begin{array}{c}31.66 \pm \\
0.57\end{array}$ & $\begin{array}{c}5.86 \pm \\
0.07\end{array}$ & $\begin{array}{c}19.56 \pm \\
0.12\end{array}$ & $\begin{array}{c}15.33 \pm \\
0.57\end{array}$ & $\begin{array}{c}20.33 \pm \\
1.15\end{array}$ & $\begin{array}{c}5.90 \pm \\
0.09\end{array}$ \\
\hline RMB-108 & $\begin{array}{c}19.91 \pm \\
0.07\end{array}$ & $\begin{array}{c}18.33 \pm \\
0.58\end{array}$ & $\begin{array}{c}33.00 \pm \\
1.00\end{array}$ & $\begin{array}{c}5.96 \pm \\
0.05\end{array}$ & $\begin{array}{c}19.68 \pm \\
0.03\end{array}$ & $\begin{array}{c}16.33 \pm \\
0.57\end{array}$ & $\begin{array}{c}21.00 \pm \\
1.00\end{array}$ & $\begin{array}{c}5.84 \pm \\
0.06\end{array}$ \\
\hline RMB-408 & $\begin{array}{c}20.14 \pm \\
0.03\end{array}$ & $\begin{array}{c}19.00 \pm \\
0.56\end{array}$ & $\begin{array}{c}30.33 \pm \\
0.57\end{array}$ & $\begin{array}{c}6.17 \pm \\
0.06\end{array}$ & $\begin{array}{c}19.85 \pm \\
0.04\end{array}$ & $\begin{array}{c}17.00 \pm \\
0.00\end{array}$ & $\begin{array}{c}19.66 \pm \\
0.57\end{array}$ & $\begin{array}{c}6.23 \pm \\
0.05\end{array}$ \\
\hline RMB-141 & $\begin{array}{c}19.65 \pm \\
0.05\end{array}$ & $\begin{array}{c}17.33 \pm \\
0.57\end{array}$ & $\begin{array}{c}33.66 \pm \\
0.57\end{array}$ & $\begin{array}{c}5.63 \pm \\
0.02\end{array}$ & $\begin{array}{c}19.43 \pm \\
0.05\end{array}$ & $\begin{array}{c}15.33 \pm \\
0.57\end{array}$ & $\begin{array}{c}21.66 \pm \\
0.57\end{array}$ & $\begin{array}{c}5.73 \pm \\
0.03\end{array}$ \\
\hline RMB-101 & $\begin{array}{c}20.21 \pm \\
0.08\end{array}$ & $\begin{array}{c}19.33 \pm \\
0.57\end{array}$ & $\begin{array}{c}31.66 \pm \\
0.57\end{array}$ & $\begin{array}{c}6.15 \pm \\
0.03\end{array}$ & $\begin{array}{c}19.77 \pm \\
0.05\end{array}$ & $\begin{array}{c}17.33 \pm \\
0.57\end{array}$ & $\begin{array}{c}20.66 \pm \\
0.57\end{array}$ & $\begin{array}{c}5.91 \pm \\
0.02\end{array}$ \\
\hline MBS-27 & $\begin{array}{c}19.74 \pm \\
0.09\end{array}$ & $\begin{array}{c}18.33 \pm \\
0.57\end{array}$ & $\begin{array}{c}31.00 \pm \\
1.00\end{array}$ & $\begin{array}{c}5.81 \pm \\
0.07\end{array}$ & $\begin{array}{c}19.41 \pm \\
0.07\end{array}$ & $\begin{array}{c}16.33 \pm \\
0.57\end{array}$ & $\begin{array}{c}19.00 \pm \\
1.00\end{array}$ & $\begin{array}{c}5.89 \pm \\
0.08\end{array}$ \\
\hline КВМB-1 & $\begin{array}{c}20.00 \pm \\
0.09\end{array}$ & $\begin{array}{c}19.66 \pm \\
0.57\end{array}$ & $\begin{array}{c}33.00 \pm \\
1.00\end{array}$ & $\begin{array}{c}6.19 \pm \\
0.05\end{array}$ & $\begin{array}{c}19.70 \pm \\
0.04\end{array}$ & $\begin{array}{c}17.66 \pm \\
0.57\end{array}$ & $\begin{array}{c}21.33 \\
1.15\end{array}$ & $\begin{array}{c}6.29 \pm \\
0.03\end{array}$ \\
\hline $\begin{array}{l}\text { Mean } \pm \\
\text { SD }\end{array}$ & $\begin{array}{c}19.94 \pm \\
0.24\end{array}$ & $\frac{18.27}{1.03} \pm$ & $\frac{31.93}{1.27} \pm$ & $\begin{array}{c}6.08 \pm \\
0.33\end{array}$ & $\begin{array}{c}19.64 \pm \\
0.22\end{array}$ & $\frac{16.27 \pm}{1.03}$ & $\frac{20.81}{1.53} \pm$ & $\begin{array}{c}6.08 \pm \\
0.29\end{array}$ \\
\hline S. Em. \pm & 0.06 & 0.32 & 0.41 & 0.04 & 0.06 & 0.32 & 0.54 & 0.04 \\
\hline $\begin{array}{l}\text { C.D@ } \\
1 \%\end{array}$ & $0.17 * *$ & $0.93 * *$ & $1.21 * *$ & $0.11 * *$ & $0.18 * *$ & $0.93 * *$ & $1.58 * *$ & $0.12 * *$ \\
\hline F value & 17.44 & 9.20 & 7.87 & 87.57 & 12.52 & 9.2 & 6.32 & 53.96 \\
\hline
\end{tabular}

Note: Values are mean of three replications, S. Em.: Standard Error of Mean, C.D @ 1\%: Critical Difference, **Significant @ 1\% 
Table.6 Comparison of cooking quality of pre-released and released mothbean varieties

\begin{tabular}{|c|c|c|c|c|c|c|c|c|}
\hline \multirow[t]{2}{*}{ Varieties } & \multicolumn{4}{|c|}{ Cooked grains without soaking } & \multicolumn{4}{|c|}{ Cooked grains with soaking } \\
\hline & $\begin{array}{c}\text { Cooked } \\
\text { weight } \\
\text { (g) }\end{array}$ & $\begin{array}{c}\text { Cooked } \\
\text { volume } \\
\quad(\mathrm{ml})\end{array}$ & $\begin{array}{c}\text { Cooking } \\
\text { time } \\
\text { (min) }\end{array}$ & $\begin{array}{c}\text { Solid loss } \\
(\%)\end{array}$ & $\begin{array}{c}\text { Cooked } \\
\text { weight } \\
\text { (g) }\end{array}$ & $\begin{array}{c}\text { Cooked } \\
\text { volume } \\
(\mathbf{m l})\end{array}$ & $\begin{array}{c}\text { Cooking } \\
\text { time } \\
\text { (min) }\end{array}$ & $\begin{array}{c}\text { Solid loss } \\
(\%)\end{array}$ \\
\hline $\begin{array}{l}\text { Pre-released } \\
\text { varieties }\end{array}$ & $\begin{array}{c}19.92 \pm \\
0.36\end{array}$ & $\begin{array}{c}17.66 \pm \\
1.11\end{array}$ & $\begin{array}{c}32.22 \pm \\
0.97\end{array}$ & $\begin{array}{c}6.43 \pm \\
0.39\end{array}$ & $\begin{array}{c}19.67 \pm \\
0.36\end{array}$ & $15.66 \pm 1.11$ & $\begin{array}{c}22.00 \pm \\
1.80\end{array}$ & $6.38 \pm 0.33$ \\
\hline $\begin{array}{l}\text { Released } \\
\text { varieties }\end{array}$ & $\begin{array}{c}19.95 \pm \\
0.20\end{array}$ & $\begin{array}{c}18.50 \pm \\
0.93\end{array}$ & $\begin{array}{c}31.83 \pm \\
1.37\end{array}$ & $\begin{array}{c}5.96 \pm \\
0.19\end{array}$ & $\begin{array}{c}19.63 \pm \\
0.16\end{array}$ & $\begin{array}{c}16.50 \pm \\
0.93\end{array}$ & $\begin{array}{c}20.37 \pm \\
1.17\end{array}$ & $\begin{array}{c}5.66 \pm \\
0.19\end{array}$ \\
\hline 't' value & NS & NS & NS & $3.40 *$ & NS & NS & NS & $3.44 *$ \\
\hline
\end{tabular}

Note: Values are mean of three replications, *Significant @ 5\%, NS-Non Significant

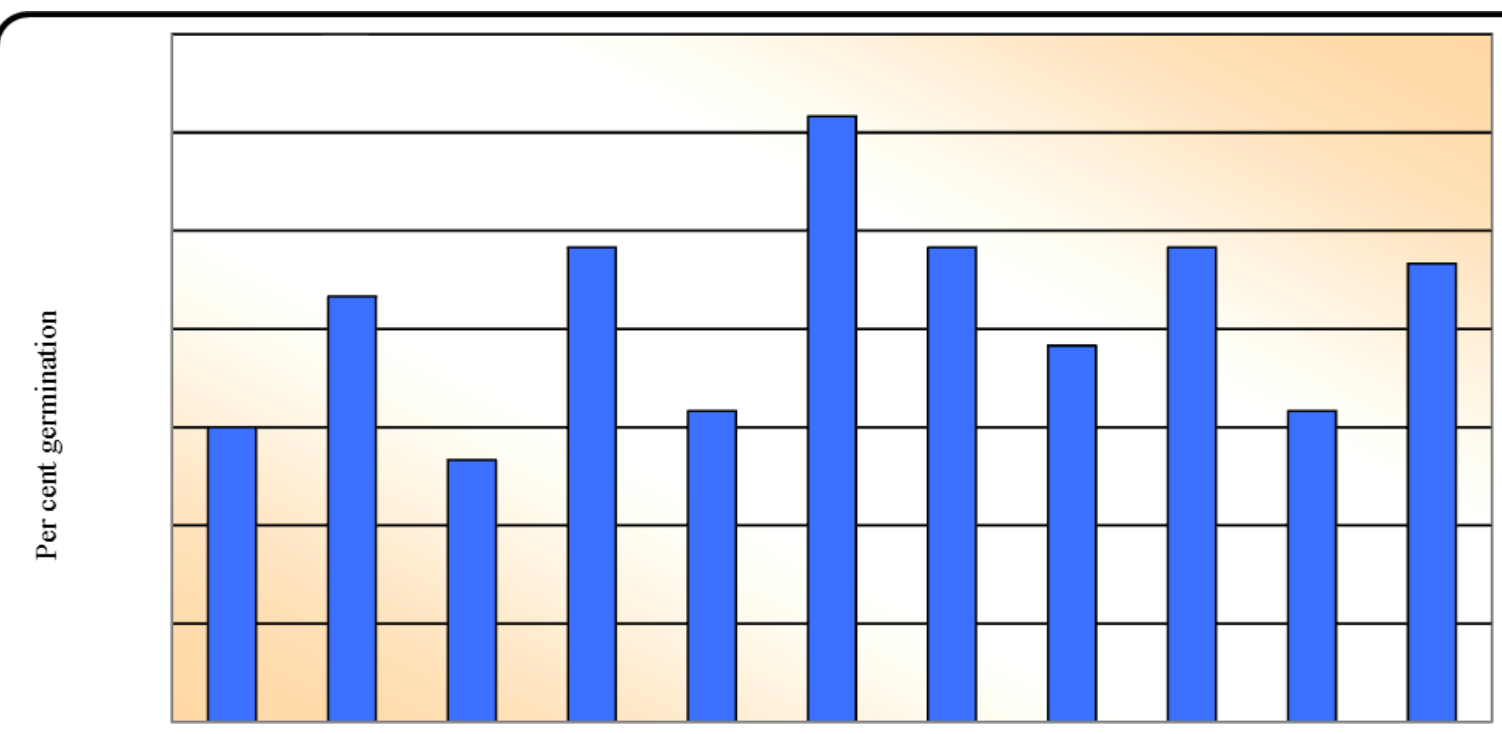

Fig. 1. Per cent germination of mothbean varieties 
Pre-released varieties had higher solid loss both in without soaking and with soaking compared to released varieties indicating that leaching of solid matter was high in prereleased varieties compared to released varieties.

Among the varieties significant differences were found with regard to physical, functional properties and cooking quality but between the pre-released and released varieties there was no significant differences found with any of the studied parameters. Hence the prereleased varieties were on par with the released varieties.

\section{Acknowledgement}

The authors are thankful to University of Agricultural Sciences, Dharwad, under SRP (STAFF RESEARCH PROJECT) for providing the financial support and to the plant breeder for providing the mothbean samples to carry out the research.

\section{References}

Barakoti, L. and Bains, K., 2007. Effect of household processing on the in vitro bioavailability of iron in mungbean (Vigna radiata). Food Nutr. Bulletin, 28(1): 18-22.
Bhokre, C. K. and Joshi, A. A., 2015. Effect of soaking on physical, functional and cooking time of cowpea, horsegram and mothbean. Food Sci. Res. J., 6(2): 357-362.

Bishnoi, S. and Khetarpaul, N., 1993. Variability in physico-chemical properties and nutrient composition of different pea cultivars. J. Food Chem., 47: 371-373.

Khattak, A. B., Bibi, N. and Aurangzeb., 2007. Quality assessment and consumer acceptability studies of newly evolved mungbean genotypes (Vigna radiata L.). American J. Food Technol., 2(6):536-542.

Mankotia, K. and Modgil, R., 2003. Effect of soaking sprouting and cooking on physico-chemical properties of mothbean (Vigna aconitifolia). J. Hum. Ecol., 14(4): 297-299.

Sareepuang, K., Siriamornpun, S., Wiset, L. and Meeso, N. 2008. Effect of soaking temperature on physical, chemical and cooking properties of parboiled fragnant rice. World J. Agri. Sci.., 4(4): 409-415.

Yadav, R. B., Khatkar, B. S. and Yadav, B. S. 2007. Morphological, physiochemical and cooking properties of some Indian rice (Oryza sativa L.) cultivars. $J$. Agri. Technol., pp. 203-210.

\section{How to cite this article:}

Pallavi Badami and Kasturiba, B. 2018. Physical, Functional Properties and Cooking Quality of Mothbean (Vigna aconitifolia (Jacq.) Marechal) Varieties. Int.J.Curr.Microbiol.App.Sci. 7(07): 3112-3119. doi: https://doi.org/10.20546/ijcmas.2018.707.363 